\title{
All-Optical Switching In A Medium of A Four-Level Vee-Cascade Atomic Medium
}

\author{
Khoa Dinh Xuan \\ Vinh University \\ Ai Nguyen Van \\ Vinh University \\ Dong Hoang Minh \\ Vinh University \\ Doai Le Van
}

Vinh University

Bang Nguyen Huy ( $\square$ bangnh@vinhuni.edu.vn )

Vinh University https://orcid.org/0000-0003-4702-3157

\section{Research Article}

Keywords: Optical bistability, Optical switches, Coherent optical communications.

Posted Date: December 13th, 2021

DOI: https://doi.org/10.21203/rs.3.rs-833664/v1

License: (c) (i) This work is licensed under a Creative Commons Attribution 4.0 International License.

Read Full License 


\title{
All-optical switching in a medium of a four-level vee-cascade atomic medium
}

\author{
Khoa Dinh Xuan ${ }^{(1)}$, Ai Nguyen Van ${ }^{(1)}$, Dong Hoang Minh ${ }^{(1,2)}$, Doai Le Van ${ }^{(1)}$, and Bang Nguyen Huy ${ }^{(1, *)}$ \\ (1) Vinh University, 182 Le Duan Street, Vinh City, Vietnam \\ (2) Ho Chi Minh City University of Food Industry, Ho Chi Minh City, Vietnam \\ *Corresponding author. E-mail: bangnh@vinhuni.edu.vn
}

\begin{abstract}
We proposed a model for all-optical switching in a medium consisting of four-level vee-cascade atomic systems excited by coupling, probe, and signal fields. It is shown that, by changing the intensity or the frequency of the signal field, the medium can be actively switched between either electromagnetically induced transparency (EIT) or electromagnetically induced absorption (EIA), which has behavior of alloptical switching. As a result, a $c w$ probe field is switched into square pulses by modulating the intensity or the frequency of the signal light. Furthermore, width of the square probe pulses can be controlled by tuning the switching period of the signal field. Such a tuneable all-optical switching is useful for finding related applications in optic communications and optical storage devices.

Keywords: Optical bistability, Optical switches, Coherent optical communications.
\end{abstract}

\section{Introduction}

All-optical switch is an important component in high-speed optical communication networks and quantum computing [1]. Over the last few decades, all-optical switching based on optical bistability in two-level atomic systems was studied. However, applications of the two-level atomic system is limited due to strong resonant absorption and only one optical field is employed for both applying and switching, thus lack of control for switching intensity thresholds. The advent of EIT [2-7] has created transparent media whose optical properties can be controlled by the external fields. Due to very steep dispersion in transparent spectral region, therefore light 
pulses can propagate with very small group velocities [8-10], the medium can achieve giant Kerr nonlinearities [11-14], and optical soliton is also easily achieved with low intensity [15-19].

In recent years, optical switching at low-light intensities based on the quantum interferences has attracted extensively attentions because of its interesting applications, such as high responsibly speed and low switching power compared with electro-optical switching and the switching of silicon waveguides or fiber-based systems. Several approaches for optical switching in EIT media were proposed theoretically and demonstrated experimentally in three-level atomic systems of lambda $(\Lambda)$, vee $(\mathrm{V})$ and ladder $(\Xi)$ configurations, such as all-optical switching based on optical bistability [20, 21] and all-optical switching in pulse mode [22-25].

In addition to the three-level systems, EIT has also been implemented in four-level atomic systems including $N$-type $(\Lambda+V)$ [26], inverted Y-type $(\Lambda+\Xi)$ [27-29], tripod-type $(\Lambda+\Lambda)$ [30] and $V+\Xi$-type [31] configurations. The advantage of four-level atomic systems is that it can be generated double-EIT windows and can be switched between EIT and EIA by signal field or external magnetic field. This provides a new way to control the light propagation between fast and slow modes [32, 33], switch between optical bistability and optical multistability [34, 35], and create optical switches. For example, Li et al., [36] studied the dynamics of pulse propagation and magneto-optic dual switching in a four-level inverted-Y atomic medium via switching on and off an external magnetic field, Gong et al., [37] presented the propagation dynamics of weak twin laser pulses and a dual-optical switching scheme in four-level semiconductor quantum dots of diamond configuration via adjusting the intensities of the control fields and the relative phase of the fields, Antón et al., [38] showed the optical bistable response and all-optical switching in a four-level tripod-type atomic system can be obtained by changing the intensity of one of the control fields, and Xiao et al., [39] experimentally demonstrated that 
an all-optical switching can be achieved by simply choosing different probe frequencies in an $\mathrm{N}$ type atom-cavity system.

Although all-optical switching in pulse mode have been studied for several four-level configurations but there still lacks study for the four-level $\mathrm{V}+\Xi$ system. As suggested by Bharti et al., [31], the four-level $\mathrm{V}+\Xi$ configuration could be actively switched between EIT and EIA modes. From the physics point of view, the medium of such behaviour is favourable to generate all-optical switching.

In this work we propose to use a four-level $\mathrm{V}+\Xi$ scheme for all-optical switching in pulse mode by numerically solving Maxwell-Bloch equations on a space-time grid. The switching of the probe laser according to the frequency and intensity of the signal laser has been considered. The advantage of this model is that we can easily switch between EIT and EIA by adjusting the frequency or intensity of the signal laser so that it is easy to create optical switching for a weak probe pulse. More, this excitation scheme can be used to excite Rydberg states having long lifetime (few $m s$ ) in which light pulse can be manipulated and slowed down to few $\mathrm{mm} / \mathrm{s}$ [33].

\section{Theoretical model}

We consider an atomic medium consists of four-level vee-cascade excitation scheme having a ground state $|1\rangle$ and three excited states $|2\rangle,|3\rangle$ and $|4\rangle$, as shown in Fig.1(a). An optical probe field with frequency $\omega_{p}$ and Rabi frequency $\Omega_{\mathrm{p}}$ drives the transition $|1\rangle \leftrightarrow|2\rangle$, while the transition $|1\rangle \leftrightarrow|3\rangle$ and $|2\rangle \leftrightarrow|4\rangle$ are excited by coupling (frequency $\omega_{c}$ ) and signal (frequency $\omega_{\mathrm{s}}$ ) optical fields with Rabi frequency $\Omega_{\mathrm{c}}$ and $\Omega_{\mathrm{s}}$, respectively. The decay rates from the states $|2\rangle$ and $|3\rangle$ to the ground state $|1\rangle$, and from the excited state $|4\rangle$ to the state $|2\rangle$ denoted by $\gamma_{21}, \gamma_{31}$ and $\gamma_{42}$, respectively. 


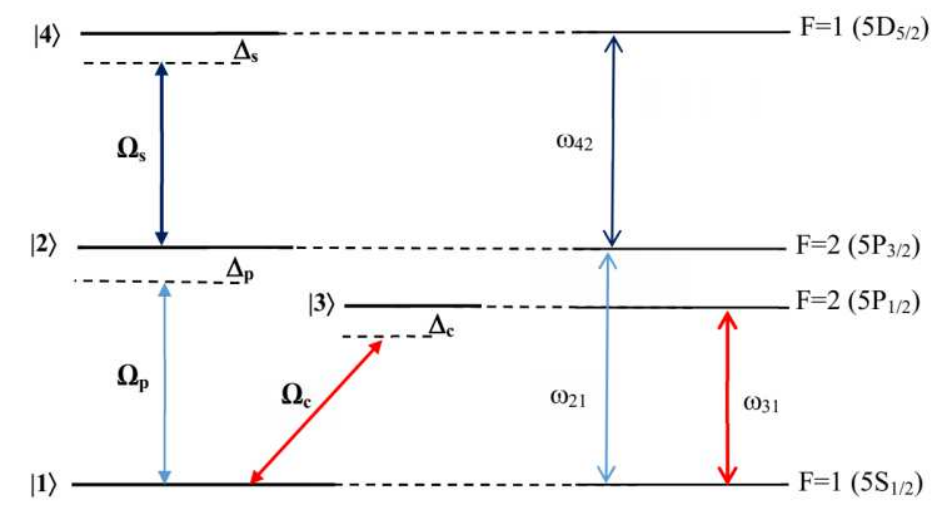

(a)

(b)

Fig. 1. (a) The four-level atomic vee-cascade scheme and (b) Relevant energy level diagram of ${ }^{87} \mathrm{Rb}$ atom.

Using the rotating-wave and the electric dipole approximations, the total Hamiltonian of the system in the interaction picture can be written as (in units $\mathrm{h}$ ).

$$
H=\left[\begin{array}{cccc}
0 & -\Omega_{p}^{*} & -\Omega_{c}^{*} & 0 \\
-\Omega_{p} & \Delta_{p} & 0 & -\Omega_{s}^{*} \\
-\Omega_{c} & 0 & \Delta_{c} & 0 \\
0 & -\Omega_{s} & 0 & \Delta_{p}+\Delta_{s}
\end{array}\right],
$$

where $\Delta_{p}=\omega_{21}-\omega_{p}, \Delta_{c}=\omega_{31}-\omega_{c}$, and $\Delta_{s}=\omega_{42}-\omega_{s}$ are frequency detunings of the probe, coupling, and signal fields, respectively. The dynamical evolution of the system can be described by the Liouville equation:

$$
\frac{\partial \rho}{\partial t}=-i[H, \rho]+\Lambda \rho
$$

where $\Lambda \rho$ represents the decaying processes. For the four-level vee-cascade system, the density matrix equations in Eq. (2) are decomposed as:

$$
\begin{aligned}
& \frac{\partial \rho_{11}}{\partial t}=\gamma_{21} \rho_{22}+\gamma_{31} \rho_{33}+i \Omega_{p}^{*} \rho_{21}-i \Omega_{p} \rho_{12}+i \Omega_{c}^{*} \rho_{31}-i \Omega_{c} \rho_{13} \\
& \frac{\partial \rho_{22}}{\partial t}=-\gamma_{21} \rho_{22}+\gamma_{42} \rho_{44}-i \Omega_{p}^{*} \rho_{21}+i \Omega_{p} \rho_{12}+i \Omega_{s}^{*} \rho_{42}-i \Omega_{s} \rho_{24} \\
& \frac{\partial \rho_{33}}{\partial t}=-\gamma_{31} \rho_{33}-i \Omega_{c}^{*} \rho_{31}+i \Omega_{c} \rho_{13}
\end{aligned}
$$




$$
\begin{gathered}
\frac{\partial \rho_{44}}{\partial t}=-\gamma_{42} \rho_{44}-i \Omega_{s}^{*} \rho_{42}+i \Omega_{s} \rho_{24}, \\
\frac{\partial \rho_{21}}{\partial t}=-\left(i \Delta_{p}+\frac{\gamma_{21}}{2}\right) \rho_{21}+i \Omega_{p}\left(\rho_{11}-\rho_{22}\right)-i \Omega_{c} \rho_{23}+i \Omega_{s}^{*} \rho_{41}, \\
\frac{\partial \rho_{31}}{\partial t}=-\left(i \Delta_{c}+\frac{\gamma_{31}}{2}\right) \rho_{31}+i \Omega_{c}\left(\rho_{11}-\rho_{33}\right)-i \Omega_{p} \rho_{32}, \\
\frac{\partial \rho_{41}}{\partial t}=-\left(i\left(\Delta_{p}+\Delta_{s}\right)+\frac{\gamma_{42}}{2}\right) \rho_{41}-i \Omega_{p} \rho_{42}-i \Omega_{c} \rho_{43}+i \Omega_{s} \rho_{21}, \\
\frac{\partial \rho_{32}}{\partial t}=-\left(i\left(\Delta_{c}-\Delta_{p}\right)+\frac{\gamma_{21}+\gamma_{31}}{2}\right) \rho_{32}-i \Omega_{p}^{*} \rho_{31}+i \Omega_{c} \rho_{12}-i \Omega_{s} \rho_{34}, \\
\frac{\partial \rho_{42}}{\partial t}=-\left(i \Delta_{s}+\frac{\gamma_{21}+\gamma_{42}}{2}\right) \rho_{42}-i \Omega_{p}^{*} \rho_{41}+i \Omega_{s}\left(\rho_{22}-\rho_{44}\right), \\
\frac{\partial \rho_{43}}{\partial t}=-\left(i\left(\Delta_{p}+\Delta_{s}-\Delta_{c}\right)+\frac{\gamma_{31}+\gamma_{42}}{2}\right) \rho_{43}+i \Omega_{c}^{*} \rho_{41}+i \Omega_{s} \rho_{23} .
\end{gathered}
$$

where the matrix elements obey conjugated and normalized conditions, namely $\rho_{i j}=\rho_{i j}^{*}(i \neq j)$, and $\rho_{11}+\rho_{22}+\rho_{33}+\rho_{44}=1$, respectively.

\section{Results and discussions}

In order to illustrate the model of all-optical switching, we apply to cold ${ }^{87} \mathrm{Rb}$ atomic medium in where the states $|1\rangle,|2\rangle,|3\rangle$ and $|4\rangle$ correspond to the levels $\left|5 \mathrm{~S}_{1 / 2}, \mathrm{~F}=1\right\rangle,\left|5 \mathrm{P}_{3 / 2}, \mathrm{~F}^{\prime}=2\right\rangle, \mid 5 \mathrm{P}_{1 / 2}$, $\left.\mathrm{F}^{\prime}=2\right\rangle,\left|5 \mathrm{D}_{5 / 2}, \mathrm{~F}^{\prime \prime}=1\right\rangle$ as depicted in Fig. 1(b). The atom and laser parameters given by [40]: $\gamma_{21}$ $=6.1 \mathrm{MHz}, \gamma_{31}=5.9 \mathrm{MHz}, \gamma_{42}=0.68 \mathrm{MHz}, \lambda_{\mathrm{p}}=780 \mathrm{~nm}, \lambda_{\mathrm{c}}=795 \mathrm{~nm}$, and $\lambda_{\mathrm{s}}=776 \mathrm{~nm}$.

\subsection{Switching between EIT and EIA}

First of all, we consider the influence of the switching signal field on the absorption and dispersion properties of medium for the probe field in the presence of coupling field at the steady regime. The linear susceptibility $\chi$ of the atomic medium for the probe light field relates to the matrix element $\rho_{21}$ determined by [5]: 


$$
\chi=2 \frac{N d_{21}}{\varepsilon_{0} E_{p}} \rho_{21}
$$

where the matrix element $\rho_{21}$ is solved numerically from set of equations (3). The imaginary $\chi^{\prime \prime}$ and real $\chi^{\prime}$ parts of the linear susceptibility represent the absorption and dispersion, respectively. In Fig. 2, we plotted the absorption $\operatorname{Im}\left(\rho_{21}\right)$ and dispersion $\operatorname{Re}\left(\rho_{21}\right)$ versus the frequency detuning $\Delta_{p}$ for the cases of absence $\left(\Omega_{\mathrm{s}}=0\right)$ and presence $\left(\Omega_{\mathrm{s}}=9 \gamma_{21}\right)$ signal field. Here we used resonant conditions $\Delta_{c}=\Delta_{s}=0$ and $\Omega_{p}=0.01 \gamma_{21}, \Omega_{c}=9 \gamma_{21}$
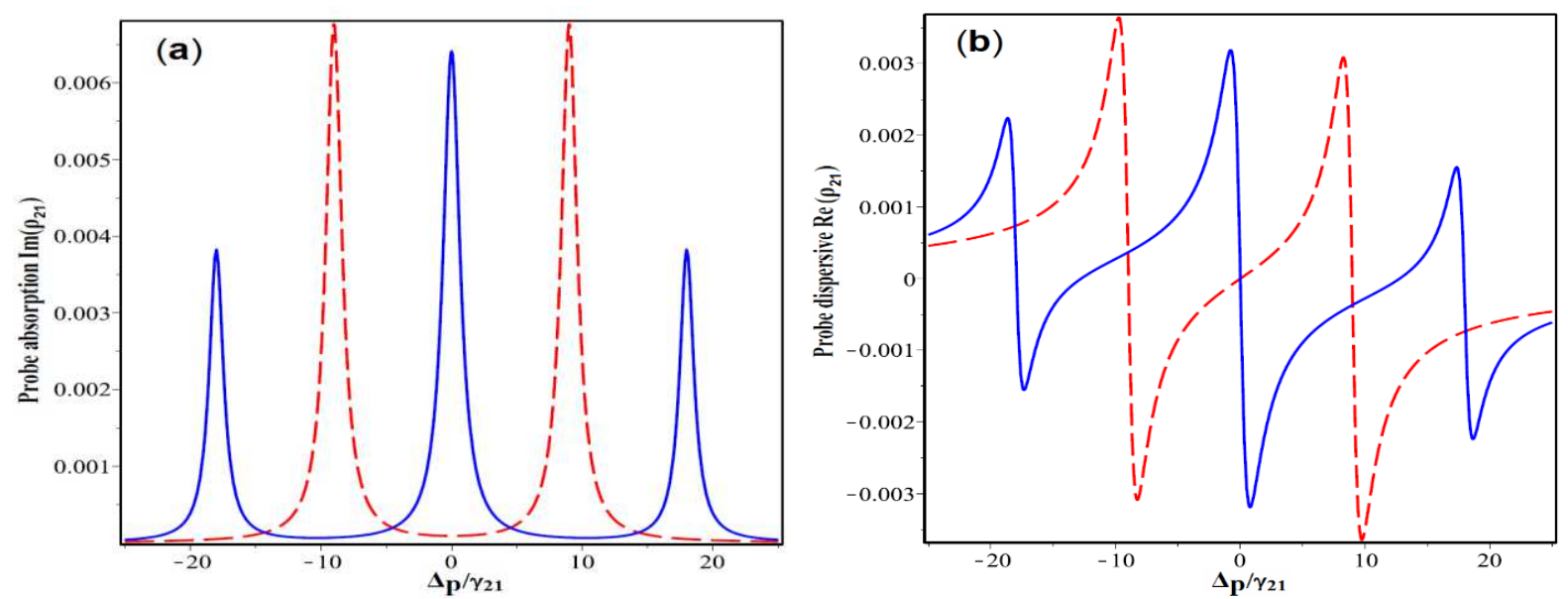

Fig. 2. Variations of probe absorption (a) and dispersion (b) versus the probe detuning $\Delta_{\mathrm{p}}$ for the given values of $\Omega_{\mathrm{p}}=0.01 \gamma_{21}, \Omega_{\mathrm{c}}=9 \gamma_{21}, \Delta_{\mathrm{c}}=0, \Delta_{\mathrm{s}}=0, \Omega_{\mathrm{s}}=9 \gamma_{21}$ when the switching field off (dashed) or on (solid) at Rabi frequency.

As shown in Fig. 2, the absorption and dispersion spectra of the probe field depend sensitively on the ON or OFF mode of the signal field. Indeed, when switching field OFF, $\Omega_{\mathrm{s}}=0$ (dashed lines), the absorption of the probe field can be suppressed thus the medium becomes completely transparent to the probe field at line center, which the corresponding dispersion profile has a positive slope near zero detuning (dashed line Fig. 2b) hence the probe light is slowed down. However, when the signal field is turns on, $\Omega_{\mathrm{s}}=\Omega_{\mathrm{c}}=9 \gamma_{21}$, the medium is switched 
from transparent to a complete absorbing mode (EIA) at the line center. Furthermore, the absorption profile for the probe field is switched from single transparency window centered at $\Delta_{\mathrm{p}}$ $=0$ to a double-transparency window located at $\Delta_{\mathrm{p}}= \pm \Omega_{\mathrm{c}}$. The corresponding dispersion profile has a negative slope near zero detuning (solid line Fig. 2b), thus the medium speeds up group velocity of the probe light.

\subsection{All-optical switching}

Now we extend our consideration from the steady to a dynamical regime. Under the slowlyvarying envelope and rotating-wave approximations, volution of the probe field is represented by the following wave equation [18]:

$$
\frac{\partial \Omega_{p}(z, t)}{\partial z}+\frac{1}{c} \frac{\partial \Omega_{p}(z, t)}{\partial t}=i \alpha \gamma_{21} \rho_{21}(z, t)
$$

here $\alpha=\frac{\omega_{p} N\left|d_{21}\right|^{2}}{4 \varepsilon_{0} c \mathrm{~h} \gamma_{21}}$ is the propagation constant. For a convenience, we represent Rabi frequency of the probe field by $\Omega_{p}(z, t)=\Omega_{p 0} f(z, t)$, where $\Omega_{p 0}$ is a real constant indicating the maximal value of the Rabi frequency at the entrance (i.e., at $\mathrm{z}=0)$, and $f(z, t)$ is a dimensionless spatiotemporal pulse-shaped function. In a moving frame with $\xi=z$ and $\tau=t-z / c$, the optical Bloch matrix equations (3a) - (3j) for the density matrix elements $\rho_{i j}(\xi, \tau)$ and Maxwell's wave equation (5) for the probe field $f(\xi, \tau)$ can be rewritten by:

$$
\begin{aligned}
& \frac{\partial \rho_{11}}{\partial \tau}=\gamma_{21} \rho_{22}+\gamma_{31} \rho_{33}+i \Omega_{p 0} f^{*}(\xi, \tau) \rho_{21}-i \Omega_{p 0} f(\xi, \tau) \rho_{12}+i \Omega_{c}^{*} \rho_{31}-i \Omega_{c} \rho_{13}, \\
& \frac{\partial \rho_{22}}{\partial \tau}=-\gamma_{21} \rho_{22}+\gamma_{42} \rho_{44}-i \Omega_{p 0} f^{*}(\xi, \tau) \rho_{21}+i \Omega_{p 0} f(\xi, \tau) \rho_{12}+i \Omega_{s}^{*} \rho_{42}-i \Omega_{s} \rho_{24}, \\
& \frac{\partial \rho_{33}}{\partial \tau}=-\gamma_{31} \rho_{33}-i \Omega_{c}^{*} \rho_{31}+i \Omega_{c} \rho_{13} \\
& \frac{\partial \rho_{44}}{\partial \tau}=-\gamma_{42} \rho_{44}-i \Omega_{s}^{*} \rho_{42}+i \Omega_{s} \rho_{24},
\end{aligned}
$$




$$
\begin{aligned}
& \frac{\partial \rho_{21}}{\partial \tau}=-\left(i \Delta_{p}+\frac{\gamma_{21}}{2}\right) \rho_{21}+i \Omega_{p 0} f(\xi, \tau)\left(\rho_{11}-\rho_{22}\right)-i \Omega_{c} \rho_{23}+i \Omega_{s}^{*} \rho_{41}, \\
& \frac{\partial \rho_{31}}{\partial \tau}=-\left(i \Delta_{c}+\frac{\gamma_{31}}{2}\right) \rho_{31}+i \Omega_{c}\left(\rho_{11}-\rho_{33}\right)-i \Omega_{p 0} f(\xi, \tau) \rho_{32}, \\
& \frac{\partial \rho_{41}}{\partial \tau}=-\left(i\left(\Delta_{p}+\Delta_{s}\right)+\frac{\gamma_{42}}{2}\right) \rho_{41}-i \Omega_{p 0} f(\xi, \tau) \rho_{42}-i \Omega_{c} \rho_{43}+i \Omega_{s} \rho_{21}, \\
& \frac{\partial \rho_{32}}{\partial \tau}=-\left(i\left(\Delta_{c}-\Delta_{p}\right)+\frac{\gamma_{21}+\gamma_{31}}{2}\right) \rho_{32}-i \Omega_{p 0} f^{*}(\xi, \tau) \rho_{31}+i \Omega_{c} \rho_{12}-i \Omega_{s} \rho_{34}, \\
& \frac{\partial \rho_{42}}{\partial \tau}=-\left(i \Delta_{s}+\frac{\gamma_{21}+\gamma_{42}}{2}\right) \rho_{42}-i \Omega_{p 0} f^{*}(\xi, \tau) \rho_{41}+i \Omega_{s}\left(\rho_{22}-\rho_{44}\right), \\
& \frac{\partial \rho_{43}}{\partial \tau}=-\left(i\left(\Delta_{p}+\Delta_{s}-\Delta_{c}\right)+\frac{\gamma_{31}+\gamma_{42}}{2}\right) \rho_{43}+i \Omega_{c}^{*} \rho_{41}+i \Omega_{s} \rho_{23}, \\
& \frac{\partial f(\xi, \tau)}{\partial(\alpha \xi)}=i \frac{\gamma_{21}}{\Omega_{p 0}} \rho_{21}(\xi, \tau) .
\end{aligned}
$$

In the following, we solve numerically the set of equations (6) on a space-time grid by using a combination of the four-order Runge-Kutta and finite difference methods which were developed from our previous work [18]. We assumed the initial condition is all atoms in the ground state $|1\rangle$ whereas the boundary condition is the probe pulse having a Gaussian-type shape $f(\xi=0, \tau)=\exp \left[-(\ln 2)(\tau-30)^{2} / \tau_{0}^{2}\right]$, with $\tau_{0}=6 / \gamma_{21}$ is the temporal width of the pulse at the entrance of the medium.

In Fig. 3 we plotted spatiotemporal evolution of intensity (square of magnitude) of the probe field at different intensities of the signal field when $\Omega_{c}=9 \gamma_{21}, \Delta_{\mathrm{c}}=\Delta_{\mathrm{s}}=\Delta_{\mathrm{p}}=0$. It is shown that the OFF or ON mode of the signal field affects sensitively on the probe pulse absorption. When the signal field OFF (i.e., $\Omega_{\mathrm{s}}=0$ ), the atomic medium is transparent to the probe pulse, namely, the probe pulse remains unchanged over a long traveling distance (Fig. 3a). As the signal field $\mathrm{ON}$ at $\Omega_{\mathrm{s}}=\Omega_{\mathrm{c}}=9 \gamma_{21}$, the probe pulse can be absorbed completely, even in a noticeably short propagation distance (see Fig. 3b). This strong absorption associates with EIA phenomenon, as 
indicated in Fig. 2a. Consequently, by switching intensity of the signal field the medium can be switched to either EIT or EIA for the probe pulse.
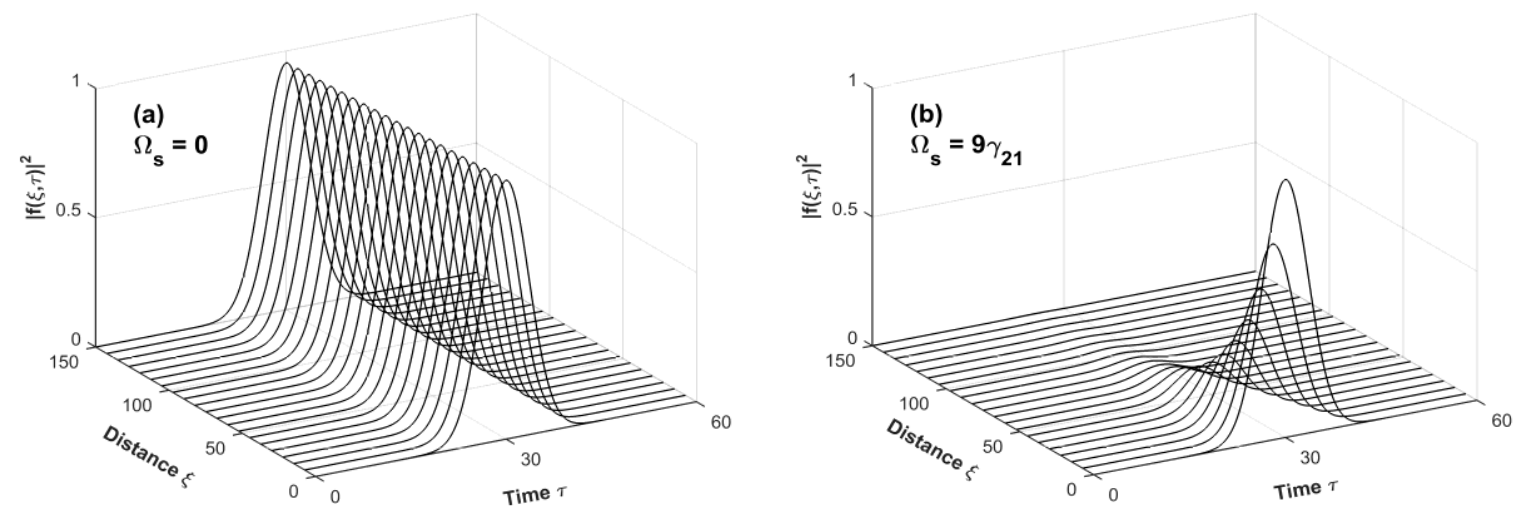

Fig. 3. Space-time evolution of the normalized probe intensity at $\Omega_{\mathrm{s}}=0$ (a) and $\Omega_{\mathrm{s}}=9 \gamma_{21}$ (b). Other used parameters are $\Omega_{0 \mathrm{p}}=0.01 \gamma_{21}, \Omega_{\mathrm{c}}=9 \gamma_{21}, \Delta_{\mathrm{p}}=\Delta_{\mathrm{c}}=\Delta_{\mathrm{s}}=0$; time $\tau$ and propagation distance $\xi$ are calculated in units of $\gamma_{21}{ }^{-1}$ and $\alpha^{-1}$, respectively.

In Fig.4, we plotted the normalized probe field intensity for different values of the signal detuning $\Delta_{\mathrm{s}}$ while keeping other parameters at $\Delta_{\mathrm{p}}=0, \Delta_{\mathrm{c}}=9 \gamma_{21}, \Omega_{0 \mathrm{p}}=0.01 \gamma_{21}, \Omega_{\mathrm{c}}=8 \gamma_{21}$, and $\Omega_{\mathrm{s}}=$ $9 \gamma_{21}$. When $\Delta_{\mathrm{s}}=0$, the medium is almost transparent for probe pulse (Fig.4a) whereas it absorbs completely probe pulses as $\Delta_{\mathrm{s}}$ increasing to a value $\Delta_{\mathrm{s}}=10 \gamma_{21}$ (see Fig. 4b). From this feature one can conclude that the medium can be switched from EIT to EIA by tuning frequency of the signal field. 

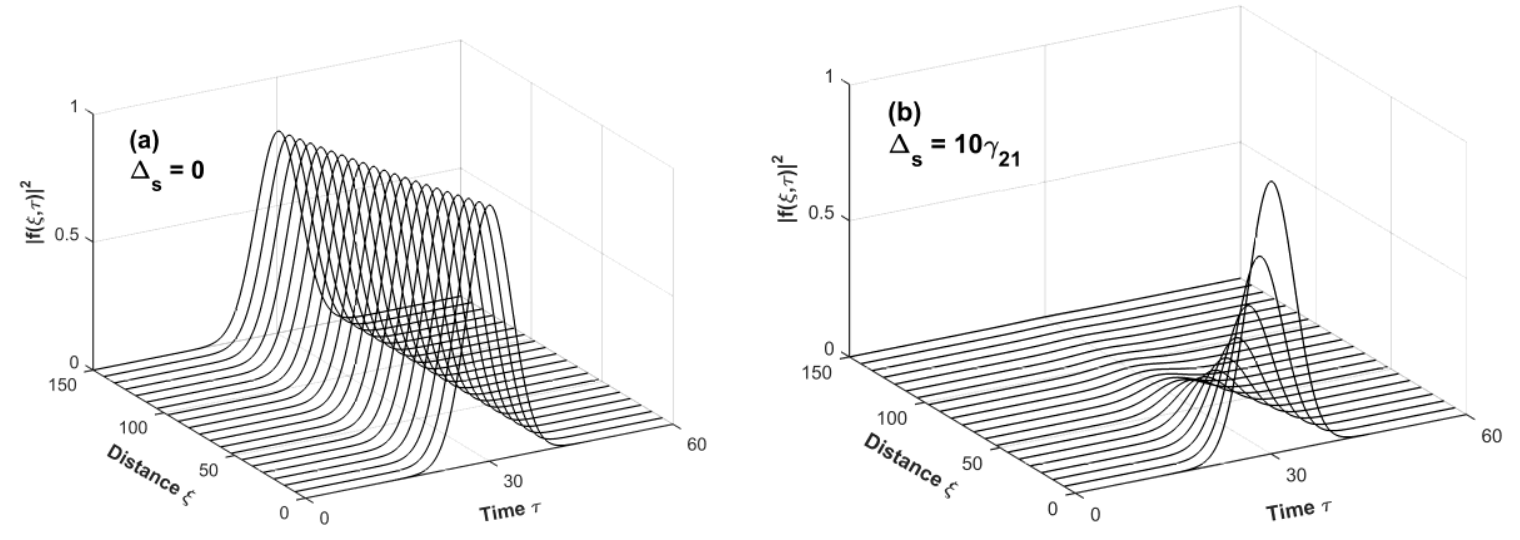

Fig. 4. The space-time evolution of normalized probe field intensity at $\Delta_{\mathrm{s}}=0$ (a) and $\Delta_{\mathrm{s}}=10 \gamma_{21}$ (b). Other parameters are $\Omega_{0 \mathrm{p}}=0.01 \gamma_{21}, \Omega_{\mathrm{c}}=8 \gamma_{21}, \Omega_{\mathrm{s}}=9 \gamma_{21}, \Delta_{\mathrm{p}}=0, \Delta_{\mathrm{c}}=9 \gamma_{21}$; time $\tau$ and propagation distance $\xi$ are calculated in units of $\gamma_{21}^{-1}$ and $\alpha^{-1}$, respectively.

Next, we consider a possible way to realize all-optical switching for the probe field by tuning parameters of the signal field, as shown in Fig.5. Here, the probe field (solid lines) is assumed to be a continuous wave $(c w)$ whereas the switching signal field (dashed lines) to be nearly-square pulses with smooth rising and falling edges. The signal field is switched by the following rules:

$$
\begin{aligned}
& \Omega_{\mathrm{s}}(\tau)=\Omega_{\mathrm{s} 0}\{1-0.5[\tanh 0.4(\tau-20)+\tanh 0.4(\tau-45)-\tanh 0.4(\tau-70)+\tanh 0.4(\tau-95)]\} \\
& \Omega_{\mathrm{s}}(\tau)=\Omega_{\mathrm{s} 0}\{1-0.5[\tanh 0.2(\tau-40)+\tanh 0.2(\tau-90)-\tanh 0.2(\tau-140)+\tanh 0.2(\tau-190)]\}
\end{aligned}
$$

Here, the intensity of the signal field in Eq. (7) or Eq. (8) is switched with approximate period 50/ $\gamma_{21}$ and 100/ $\gamma_{21}$, respectively. In Fig.5 we plotted the switching field when the amplitude of the signal field is normalized by its peak value $\Omega_{\mathrm{s} 0}=9 \gamma_{21}$. Fig. 5 shows that the switching periods of both probe and signal fields are the same. Furthermore, the probe transmission is switched to the ON or OFF mode when the intensity of the signal field is OFF or ON, respectively. On the other hand, oscillations at the front edge of the probe pulse can be extinguished when increasing width of the signal pulse (Fig. 5b). 

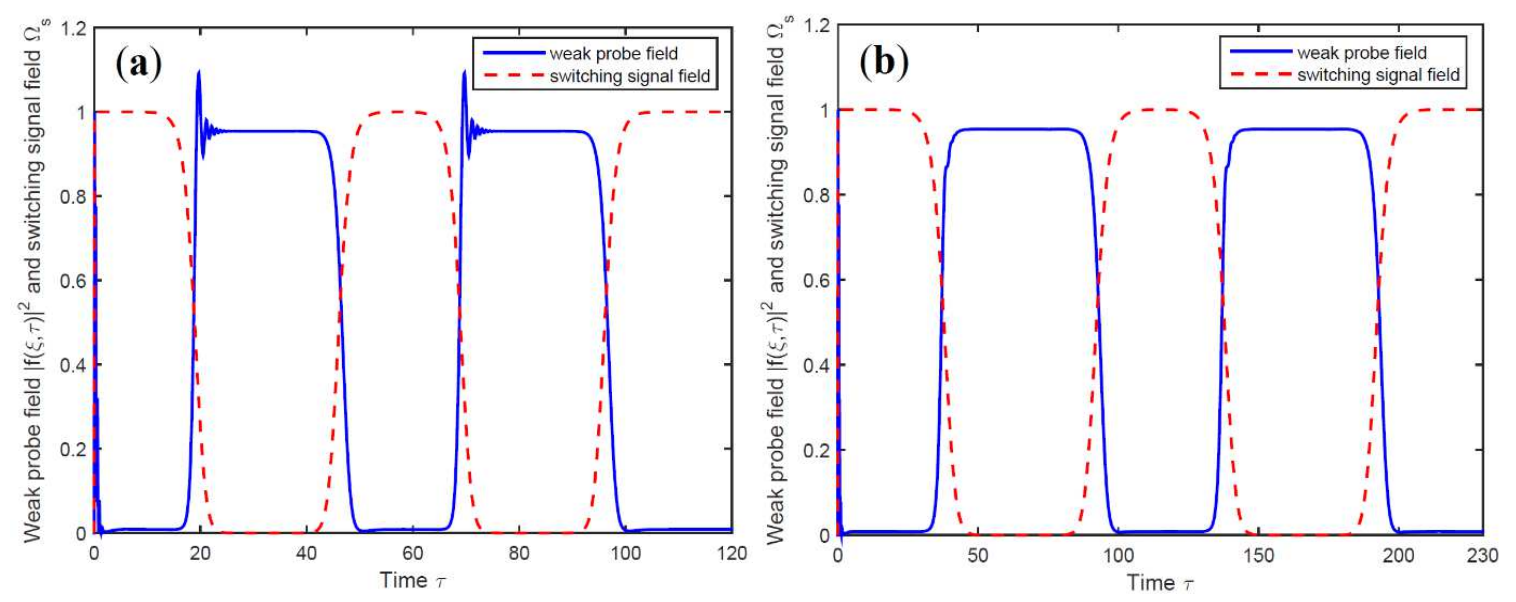

Fig. 5. Time evolution of a $c w$ probe field (solid line) at $\xi=150 / \alpha$ when the signal field (dashed lines) switched at approximate period $50 / \gamma_{21}$ (a) and $100 / \gamma_{21}$ (b). Other parameters are $f(\xi=0, \tau)=1, \Omega_{\mathrm{p} 0}=$ $0.01 \gamma_{21}, \Omega_{\mathrm{s} 0}=\Omega_{\mathrm{c}}=9 \gamma_{21}, \Delta_{\mathrm{p}}=0, \Delta_{\mathrm{c}}=0, \Delta_{\mathrm{s}}=0$; time $\tau$ is calculated in units of $\gamma_{21}{ }^{-1}$.

In Fig.6, we consider the dynamics of the $c w$ probe field (solid lines) under modulating frequency of the signal field (dashed lines). Here, frequency detuning of the probe field is chosen as a nearly-square pulse with smooth rising and falling edges as follows:

$$
\begin{gathered}
\Delta_{\mathrm{s}}(\tau)=\Delta_{\mathrm{s} 0}\{1-0.5[\tanh 0.4(\tau-20)+\tanh 0.4(\tau-45)-\tanh 0.4(\tau-70)+\tanh 0.4(\tau-95)]\}, \\
\Delta_{\mathrm{s}}(\tau)=\Delta_{\mathrm{s} 0}\{1-0.5[\tanh 0.2(\tau-40)+\tanh 0.2(\tau-90)-\tanh 0.2(\tau-140)+\tanh 0.2(\tau-190)]\} .
\end{gathered}
$$

Here, the frequency detuning of the signal field in Eq. (9) or Eq. (10) is switched with approximate period $50 / \gamma_{21}$ and $100 / \gamma_{21}$, respectively. In both cases, the frequency detuning is normalized by its peak value as $\Delta_{\mathrm{s} 0}=10 \gamma_{21}$. We can see that the probe transmission is switched to either ON or OFF when the frequency detuning of the signal field is switched OFF or ON, respectively. As indicated in the Sect. 3.1, the ON or OFF mode for probe field can be attributed from EIT or EIA mode of the medium, respectively. 

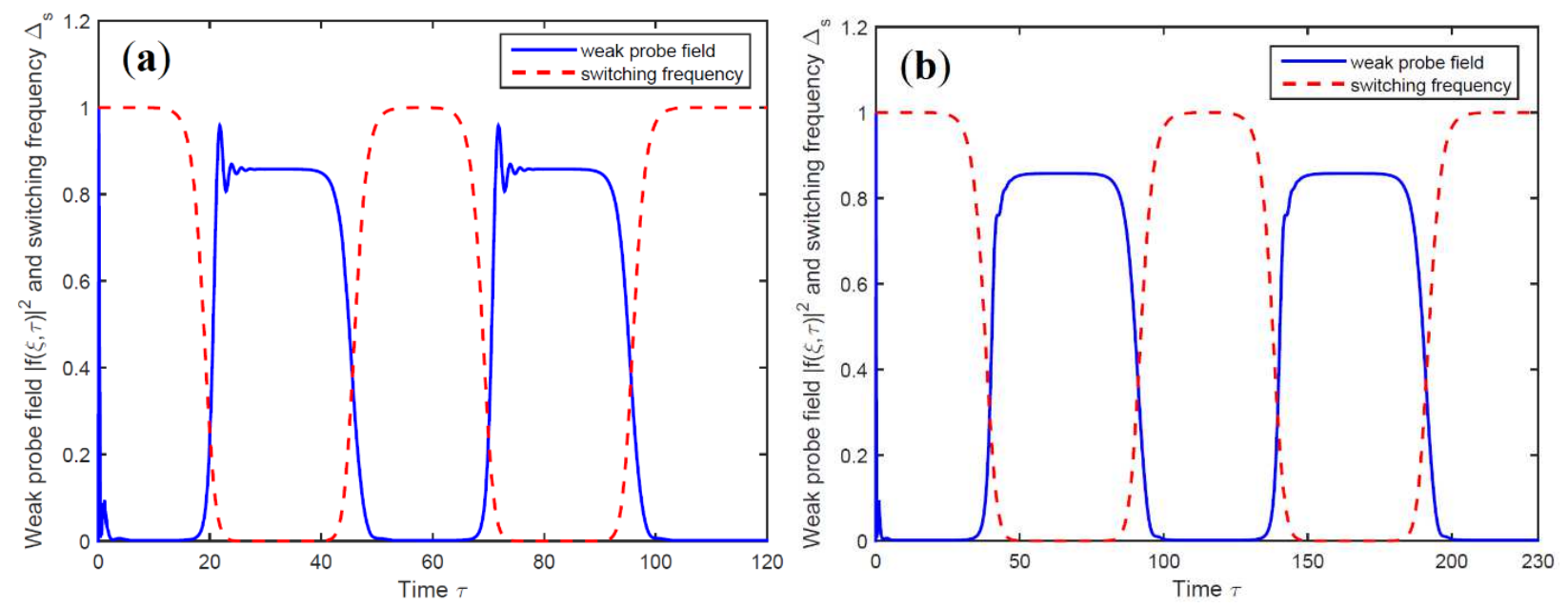

Fig. 6. Time evolution of a $\mathrm{cw}$ probe field (solid line) at $\xi=150 / \alpha$ when the signal field (dashed lines) switched at approximate period $50 / \gamma_{21}$ (a) and $100 / \gamma_{21}$ (b). Other parameters are $f(\xi=0, \tau)=1, \Omega_{\mathrm{p} 0}=$ $0.01 \gamma_{21}, \Omega_{\mathrm{c}}=8 \gamma_{21}, \Omega_{\mathrm{s}}=9 \gamma_{21}, \Delta_{\mathrm{p}}=0, \Delta_{\mathrm{c}}=9 \gamma_{21}, \Delta_{\mathrm{s} 0}=10 \gamma_{21}$; time $\tau$ is calculated in units of $\gamma_{21}{ }^{-1}$.

Finally, we consider the behavior of optical switching according as intensity (Fig.7) and the frequency (Fig. 8) of the signal field in strong probe regime. Fig.7 and Fig.8 show the cases of Fig.5(b) Fig.6(b), respectively, while the probe intensity is increased to $\Omega_{\mathrm{p} 0}=0.5 \gamma_{21}$ (a) and $\Omega_{\mathrm{p} 0}=1 \gamma_{21}$ (b). From these figures we can see that when intensity of the probe field is of the same order with the coupling field, the switching efficiency decreases. To explain the phenomenon, we plot spatiotemporal evolution of intensity of the probe pulse at $\Omega_{\mathrm{s}}=\Omega_{\mathrm{c}}=9 \gamma_{21}, \Delta_{\mathrm{c}}=\Delta_{\mathrm{s}}=\Delta_{\mathrm{p}}=0$ and $\Omega_{\mathrm{p} 0}=0.5 \gamma_{21}$ (a) and $\Omega_{\mathrm{p} 0}=1 \gamma_{21}$ (b) as shown in Fig.9. Due to strong intensity of the probe field, its intensity will not be absorbed completely, then it may transfer to the next period, which results higher background (Fig.7b and Fig.8b), namely lower switching efficiency. Moreover, the strong probe pulse corresponding to self-phase modulation is formed and the switching process can be broken down gradually. 

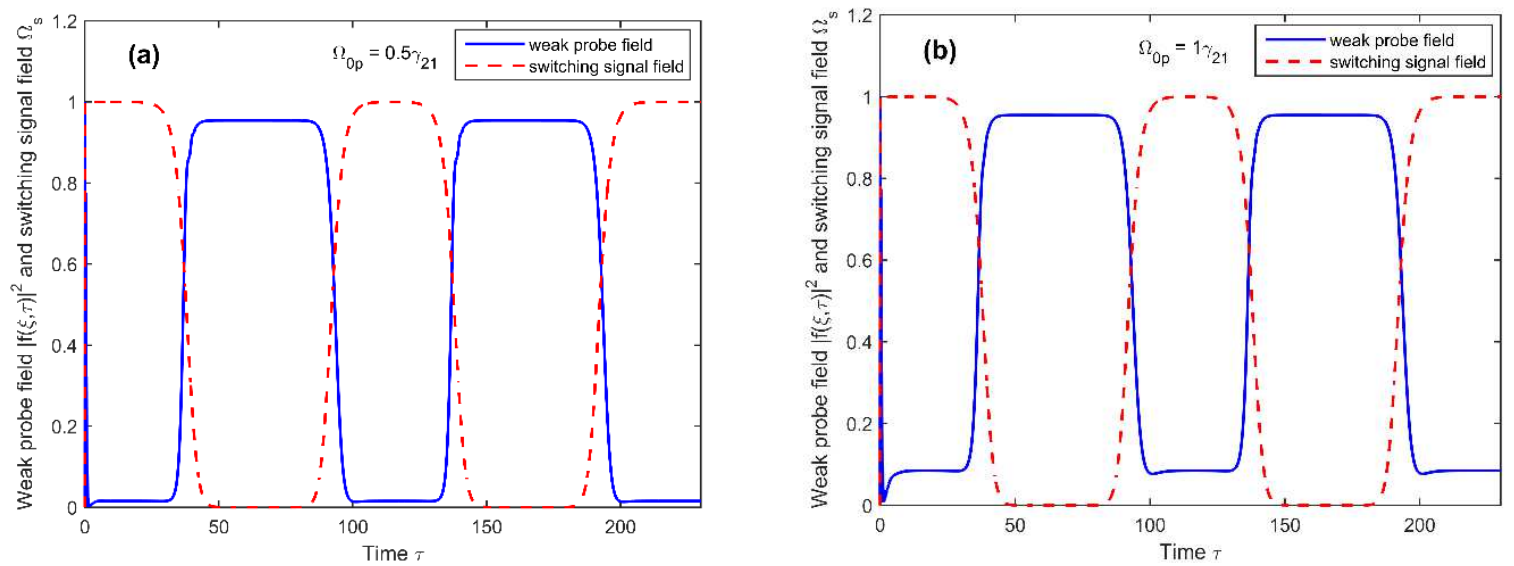

Fig. 7. Time evolution of a $c w$ probe field (solid line) at $\xi=150 / \alpha$ when the signal field (dashed lines) switched at approximate period $100 / \gamma_{21}$, and the probe field intensity $\Omega_{\mathrm{p} 0}=0.5 \gamma_{21}$ (a) and $\Omega_{\mathrm{p} 0}=1 \gamma_{21}$ (b). Other parameters given as same as those in Fig.5.
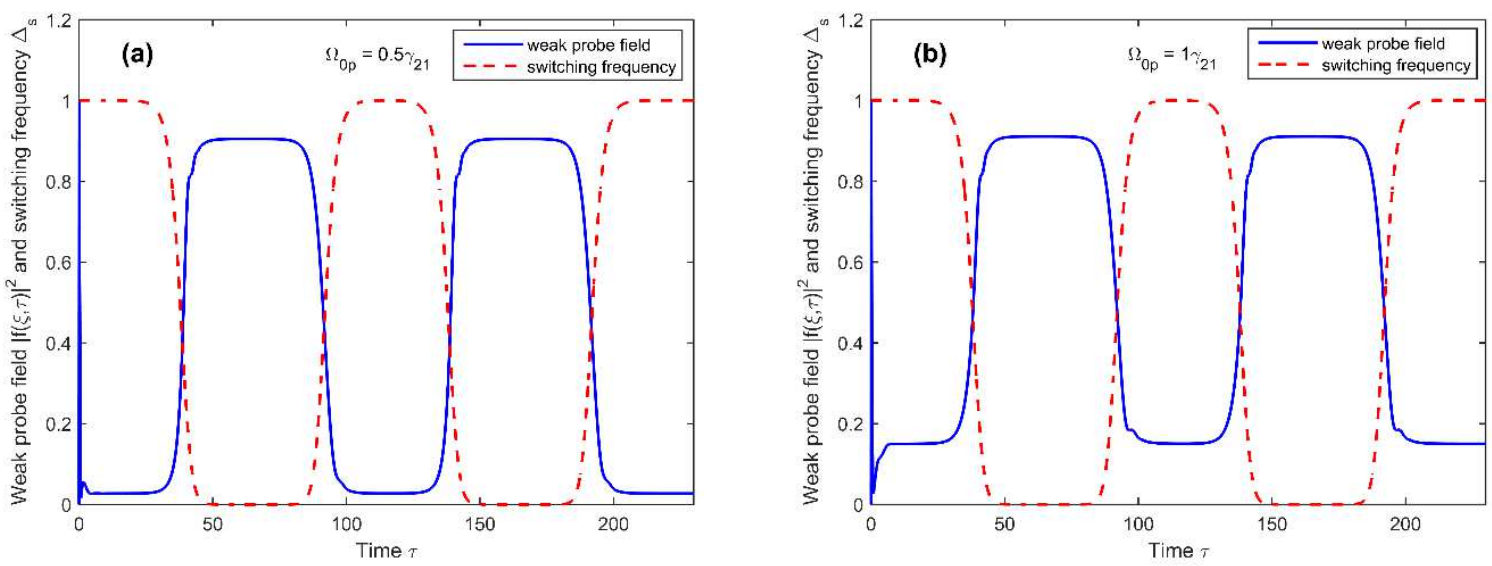

Fig. 8. Time evolution of a cw probe field (solid line) at $\xi=150 / \alpha$ when the signal field (dashed lines) switched at approximate period $100 / \gamma_{21}$, and the probe field intensity $\Omega_{\mathrm{p} 0}=0.5 \gamma_{21}$ (a) and $\Omega_{\mathrm{p} 0}=1 \gamma_{21}$ (b). Other parameters given as same as those in Fig.6. 

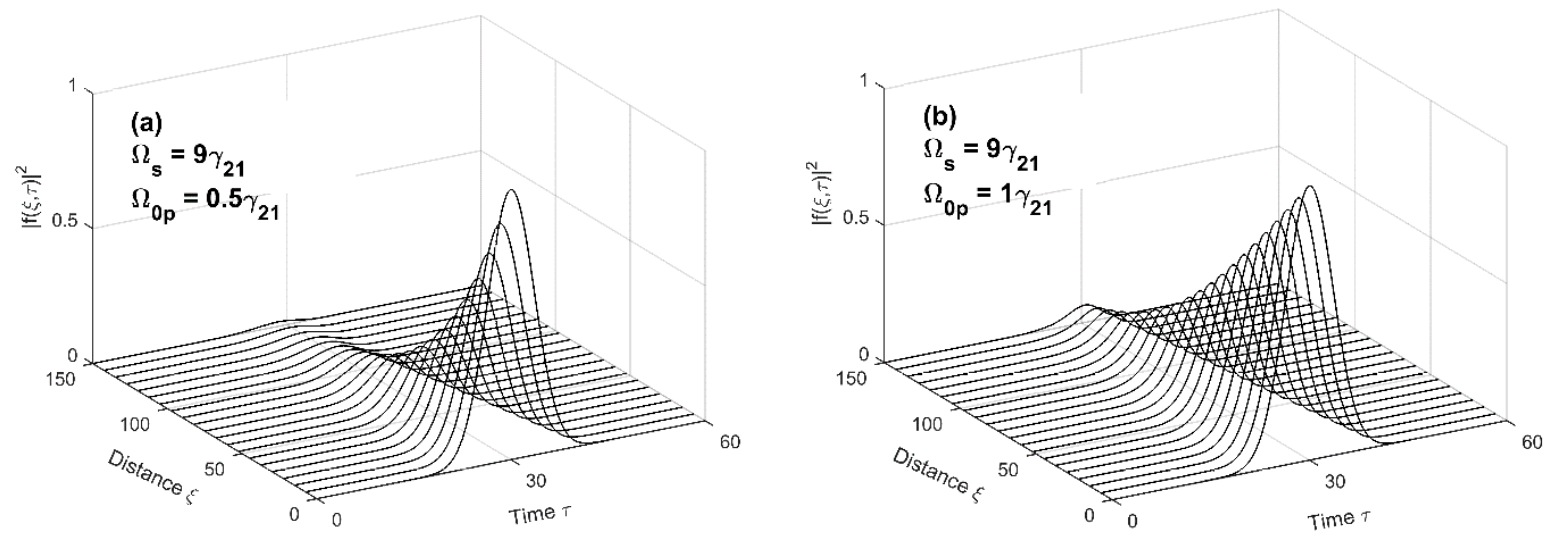

Fig. 9. Space-time evolution of the normalized probe intensity at $\Omega_{\mathrm{s}}=\Omega_{\mathrm{c}}=9 \gamma_{21}$, and $\Omega_{0 \mathrm{p}}=0.5 \gamma_{21}$ (a) and $\Omega_{0 \mathrm{p}}=1 \gamma_{21}$ (b). Other parameters given as same as those in Fig.3.

\section{Conclusion}

We have proposed a model of four-level vee-cascade atomic medium to realize all-optical switching for a probe light in weak and strong probe regimes. Due to quantum interferences among atomic transition paths induced by coupling, probe and signal optical fields, the medium can be switched to either EIT or EIA regime by switching the signal field to OFF or ON, respectively. As a result, an all-optical switching can be generated in which the $c w$ probe field is switched into pulses by modulating intensity or frequency of the signal light. Furthermore, width of the probe pulses can be controlled by tuning switching period of the signal field. Such a tuneable all-optical switching is useful for finding related applications in optics communications and optical storage devices.

Acknowledgements: The financial support from Vietnamese National Foundation of Science and Technology Development under Grant No. 103.03-2017.332 and Ministry of Education and Training under Grant No. B2018-TDV-01SP is acknowledged. 


\section{References}

[1] Ishikawa H, Ultrafast All-Optical Signal Processing Devices, John Wile \& Sons, Singapore, 2008.

[2] Imamoğlu A and Harris S E 1989, "Lasers without inversion: interference of dressed lifetime broadened states", Opt. Lett. 14 1344-1346

[3] Boller K J, Imamoglu A, Harris S E 1991, Observation of electromagnetically induced transparency, Phys. Rev. Lett. 662593.

[4] Fleischhauer M, Imamoglu A, Marangos J P 2005, Electromagnetically induced transparency: optics in coherent media, Rev. Mod. Phys. 77633.

[5] Doai L V, Trong P V, Khoa D X, and Bang N H 2014, Electromagnetically induced transparency in five-level cascade scheme of ${ }^{85} \mathrm{Rb}$ atoms: An analytical approach, Optik 125 3666.

[6]Khoa D X, Trong P V, Doai L V, and Bang N H 2016, Electromagnetically induced transparency in a five-level cascade system under Doppler broadening: an analytical approach, Phys. Scr. 91035401.

[7] Khoa D X, Trung L C, Thuan P V, Doai L V and Bang N H 2017, Measurement of dispersive profile of a multi-window EIT spectrum in a Doppler-broadened atomic medium, J. Opt. Soc. Am. B. 341255.

[8] Hau L V, Harris S E, Dutton Z, Bejroozi C H 1999, Light speed reduction to 17 metres per second in an ultracold atomic gas, Nature 397594.

[9]Anh N T, Doai L V, Son D H and Bang N H 2018, Manipulating multi-frequency light in a five-level cascade EIT medium under Doppler broadening, Optik 171 721-727. 
[10] Anh N T, Doai L V, and Bang N H 2018, Manipulating multi-frequency light in a fivelevel cascade-type atomic medium associated with giant self-Kerr nonlinearity, J. Opt. Soc. Am. B 351233.

[11] Wang H, Goorskey D and Xiao M 2001, Enhanced Kerr Nonlinearity via Atomic Coherence in a Three-Level Atomic System, Phys. Rev. Lett., 87073601.

[12] Khoa D X, Doai L V, Son D H and Bang N H 2014, Enhancement of self-Kerr nonlinearity via electromagnetically induced transparency in a five-level cascade system: an analytical approach, J. Opt. Soc. Am. B. 311330.

[13] Hamedi H R, Gharamaleki A H, and Sahrai M 2016, Colossal Kerr nonlinearity based on electromagnetically induced transparency in a five-level double-ladder atomic system, Appl. Opt 225892.

[14] Doai L V, An N L T, Khoa D X, Sau V N and Bang N H 2019, Manipulating giant crossKerr nonlinearity at multiple frequencies in an atomic gaseous medium, J. Opt. Soc. Am. B 36 2856.

[15] Huang G, Jiang K, Payne M G and Deng L 2006, Formation and propagation of coupled ultraslow optical soliton pairs in a cold three-state double- $\Lambda$-system, Phys. Rev. E 73056606.

[16] Si L G, Lu X Y, Hao X and Li J H 2010, Dynamical control of soliton formation and propagation in a Y-type atomic system with dual ladder-type electromagnetically induced transparency, J. Phys. B: At. Mol. Opt. Phys. 43065403.

[17] Chen Y, Bai Z, and Huang G 2014, Ultraslow optical solitons and their storage and retrieval in an ultracold ladder-type atomic system, Phys. Rev. A $\mathbf{8 9} 023835$. 
[18] Khoa D X, Dong H M, Doai L V and Bang N H 2017, Propagation of laser pulse in a three-level cascade inhomogeneously broadened medium under electromagnetically induced transparency conditions, Optik 131497.

[19] Dong H M, Doai L V, Khoa D X, and Bang N H 2018, Pulse propagation in an atomic medium under spontaneously generated coherence, incoherent pumping, and relative laser phase, Opt. Commun. 426 553-557.

[20] Wang H, Goorskey D and Xiao M 2002, Controlling light by light with three-level atoms inside an optical cavity, Opt. Lett., 27 1354-356.

[21] Jafarzadeh H 2017, All-optical switching in an open V-type atomic system, Laser Phys. 27025204.

[22] Schmidt H, Ram R J 2000, All-optical wavelength converter and switch based on electromagnetically induced transparency, Appl. Phys. Lett. 763173.

[23] Fountoulakis A, Terzis A F, Paspalakis E 2010, All-optical modulation based on electromagnetically induced transparency, Phys. Lett. A 3743354.

[24]Dong H M, Nga L T Y and Bang N H 2019, Optical switching and bistability in a degenerated two-level atomic medium under an external magnetic field, Appl. Opt., 584192.

[25]Dong H M and Bang N H 2019, Controllable optical switching in a closed-loop three-level lambda system, Phy. Scr. 94115510.

[26] Islam K, Bhattacharyya D, Ghosh A, Biswas D and Bandyopadhyay A 2017, Study on probe field propagation in the presence of control and coupling fields through a four-level Ntype atomic system, J. Phys. B: At. Mol. Opt. Phys. 50215401.

[27] Joshi A and Xiao M 2003, Electromagnetically induced transparency and its dispersion properties in a four-level inverted-Y atomic system, Phys. Lett. A 317370. 
[28] Joshi A and Xiao M 2004, Vacuum Rabi splitting for multilevel electromagnetically induced transparency system, Eur. Phys. J. D 30431.

[29] Joshi A and Xiao M 2005, Generalized dark-state polaritons for photon memory in multilevel atomic media, Phys. Rev. A 71041801.

[30] Yang X, Li S, Zhang C and Wang H 2009, Enhanced cross-Kerr nonlinearity via electromagnetically induced transparency in a four-level tripod atomic system, J. Opt. Soc. Am. B 261423.

[31] Bharti V, Natarajan V 2015, Study of a four-level system in vee + ladder configuration, Opt. Commun. 356510.

[32] Asadpour S H 2017, Goos-Hänchen shifts due to spin-orbit coupling in the carbon nanotube quantum dot nanostructures, Appl. Opt. 56 2201-2208.

[33] Vineet Bharti, Vasant Natarajan 2017, Sub- and super-luminal light propagation using a Rydberg state, Opt. Comm. 392 180-184

[34] Asadpour S H and Soleimani H R 2016, Phase dependence of optical bistability and multistability in a four-level quantum system near a plasmonic nanostructure, J. App. Phys. 119023102.

[35] Asadpour S H and Soleimani H R 2016, Phase dependence of optical bistability and multistability in graphene nanostructure under external magnetic field, Las. Phys. Lett. 13 015204

[36] Li J, Yu R, Si L and Yang X-X 2010, Propagation of twin light pulses under magnetooptical switching operations in a four-level inverted-Y atomic medium, J. Phys. B: At. Mol. Opt. Phys. 43065502. 
[37] Qi Y, Zhou F, Yang J, Niu Y and Gong S 2013, Controllable twin laser pulse propagation and dual-optical switching in a four-level quantum dot nanostructure, J. Opt. Soc. Am. B . 30, 1928.

[38] Antón M A, Calderón O G, Melle S, Gonzalo I, Carreño F 2006, All-optical switching and storage in a four-level tripod-type atomic system, Opt. Commun. 268146.

[39] Sheng J, Yang X-H, Khadka U and Xiao M 2011, All-optical switching in an N-type foul-level atom-cavity system, Opt. Express 19(18) 17059-64.

[40] D. Steck, ${ }^{87} \mathrm{Rb}$ D line data, http://steck.us/alkalidata. 